$\begin{array}{ll}\text { Italique } & \text { Italique } \\ \text { Poésie italienne de la Renaissance }\end{array}$

IX 2006

Varia

\title{
Prefazione a un repertorio metrico della canzone italiana dai siciliani al Tasso
}

\section{Guglielmo Gorni}

\section{(2) OpenEdition}

\section{Journals}

\section{Edizione digitale}

URL: http://journals.openedition.org/italique/110

DOI: 10.4000/italique. 110

ISSN: 1663-4438

\section{Editore}

Librairie Droz

\section{Edizione cartacea}

Data di pubblicazione: 1 giugno 2006

Paginazione: 101-130

ISBN: 2-600-01088-2

ISSN: 1423-3983

\section{Notizia bibliografica digitale}

Guglielmo Gorni, «Prefazione a un repertorio metrico della canzone italiana dai siciliani al Tasso », Italique [Online], IX | 2006, online dal 31 décembre 2009, consultato il 19 avril 2019. URL : http:// journals.openedition.org/italique/110; DOI : 10.4000/italique.110 
Guglielmo Gorni

PREFAZIONE A U N

REPERTORIO METRICO

DELLA CA N Z N E ITALIANA

D A I SICILIA I A L TASO 

quant'al mondo si tesse, opra d'aragna vede

(Petrarca, Rvf. I73, 6-7) 



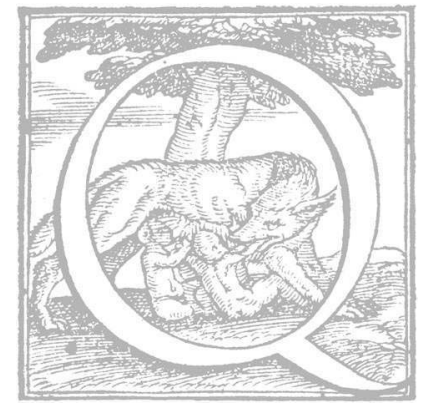

Uella dei Malavoglia, come tutti sanno, è la «casa del nespolo». Ma quanti sono in grado di distinguere a prima vista un nespolo da un corbezzolo o da un sambuco? Non dico che il candido lettore debba riconoscere, come il Manzoni sapeva fare infallibilmente, «in un campo mal coltivato [...] un bel lapazio» o additare, come era ovvio per il Pascoli, un'umile myrica nella flora circostante.

Quanti filologi hanno preso in mano con cognizione di causa una manzoniana «acetosella» o una pascoliana e dannunziana «tamerice»? E quanti lettori di Gadda, me compreso, scorrendo il Giornale di guerra e di prigionia possono dire di aver chiare nozioni di artiglieria, di come - poniamo esplodono una granata o uno shrapnel? Recensendo il secondo volume delle Opere di Gadda edite da Garzanti, per cavarmela tra obici e spolette, auspicai l'avvento di una filologia delle "parasanghe», indimenticabile relitto della lettura ginnasiale dell'Anabasi di Senofonte: un'idea che non spiacque all'amico Giancarlo Roscioni. Mi è sempre rimasto il complesso del «nespolo» e delle "parasanghe».

La metrica, e specialmente la morfologia della canzone, mi è sembrata, nei primi anni degli studi di lettere, misteriosa e intricata come la vigna di Renzo. Era ignoranza mia, ne convengo. Forse altri, più provveduto di me, sapeva del «lapazio» e della varia fortuna della fronte $\mathrm{ABbCABbC}$ nelle canzoni «distese». Comunque sia, precocemente volli riscattarmi da quell'ignoranza e percio raccolsi tutti gli schemi metrici che potevo, allo scopo d'individuarvi qualche tratto meno scostante, parametri non generici di riconoscibilità. Accumulai schede su schede, per curiosità mia e a mio profitto. E in pochi anni raccolsi un cospicuo materiale, che ora viene messo a disposizione del pubblico, per esortazione di vari amici che se ne sono serviti in maniera confidenziale o erano al corrente dell'esistenza del mio regesto.

Il volume a cui la presente prosa fa da premessa non è altro che l'edizione di uno schedario privato, vivente l'autore. Alla natura molto personale del Repertorio allude anche il sottotitolo (Repertorio metrico della canzone italiana dalle Origini al Cinquecento [REMCI]. Censimento di Guglielmo Gorni, edito per cura sua e di Massimo Malinverni), che menziona espressamente il "Censimento» da cui esso nasce. La pubblicazione, non programmata allora, si accompagna a una serie di confessioni e precisazioni per l'aspetto inconsueto del tutto, a dispetto della revisione operata. Scherzando, ero solito definire quelle centinaia di schede le mie Carte Bilancioni. Sennonché le carte dell'avvocato e bibliofilo romagnolo Pietro Bilancioni (I808-1877) erano uscite postume. Carlo e Lodovico Frati, che 
stamparono l'Indice della Parte prima (la sola uscita) nel «Propugnatore», dal I889 al I893, potevano farsi schermo del dovere di rispettare il documento e le sue incoerenze: omissioni e imprecisioni erano da mettere sul conto di un autore che non poteva più intervenire, e che però aveva ben meritato della bibliografia delle rime antiche. La parte buona del suo lavoro, quali che fossero lacune e imperfezioni, era considerata utile e degna di stampa dagli specialisti. Anche al sentimento di chi scrive, tanto mutato rispetto a quello di allora e animato da interessi in gran parte diversi, il presente Repertorio appare come un'opera postuma. Questa scusa peraltro può rivelarsi insufficiente rispetto alle attese e al rigore che l'opera impone. E stato giusto fare «come se»? Perché resta il fatto, incontestabile, che il Repertorio Metrico della Canzone Italiana, dalle Origini al Cinquecento - del quale si propone, per le citazioni correnti, l'acronimo REMCI - è la traduzione a stampa, da supporto informatico, di un ingente censimento su schede compilate a mano dallo scrivente nel corso degli anni e proposto nel volume citato con qualche integrazione e ritocco. Messo insieme sibi et amicis, come talora si legge nei codici. Il lavoro di schedatura, che ha assunto via via ambizioni di sistematicità, era cominciato al tempo (novembre 1968) della mia tesi di laurea, che verteva su altro argomento che la metrica, ed è continuato con particolare intensità nei miei anni fiorentini, dal 1969 al 1977. Ci fu, in tempi successivi, l'addizione di nuove schede e di nuovi incipit a schede già presenti, secondo il ritmo occasionale delle letture, dai Siciliani al Tasso. Questi due capisaldi cronologici restarono immutati, parendomi che la morfologia della canzone italiana, dopo il Chiabrera, assumesse un'altra fisionomia, non equiparabile a quella della tradizione precedente; anche se, di fatto, sulle schede trovarono posto a intermittenza canzoni, che si sono omesse nella stampa, di autori del Sette e perfino dell'Ottocento, come il Carducci degli Juvenilia, ligio alla tradizione.

Non è inopportuno rivelare che a una schedatura analoga, pur meno estesa, vennero da me sottoposte in quel decennio anche le ballate, che trovarono poi in Linda Pagnotta una descrittrice eccellente nel Repertorio metrico edito da Ricciardi nel 1995. E schedai pure le parole rima di tutte le sestine che potei raccogliere: un progetto che mi pare di perdurante interesse e che sarebbe da riprendere con miglior metodo e lena (vi attende ora, con programmatica diligenza, Andrea Comboni). Ma era soprattutto la morfologia della canzone a interessarmi, e ad essa rivolsi, per quanto potevo, costanti cure. Il primo lavoro che presupponeva la presenza e la fruizione di un vasto censimento fu il contributo per la Miscellanea pavese in onore di Carlo Dionisotti. ${ }^{2}$ Mi è grato ricordare come Dionisotti, presentandosi il volume edito in suo onore nell'Aula Foscolo dell'Università di Pavia, salutasse con parole d'incoraggiamento quella che a lui pareva una rinascita degli studi metrici e in particolare 
morfologici, non più di moda dai tempi del Biadene e del Flamini e adibiti semmai a una lettura stilistica: in servizio della critica, piuttosto che della filologia in senso stretto, come era chiaro nelle «lezioni sulle forme metriche» di Mario Fubini (1962, 1970'). Mi sentivo l'epigono di una tradizione di ricerche che era, come spiritosamente scrisse d'Arco Silvio Avalle in Preistoria dell'endecasillabo (Milano-Napoli, Ricciardi, 1963), un «ramo secco» degli studi letterari. Un ramo destinato, come si vide poi, a miracolosa, inopinata fioritura.

Schedai le canzoni stampate in antologie o in sillogi d'autore; ripresi in mano edizioni di antichi canzonieri, come il quattrocentesco codice Isoldiano, le cui rime furono pubblicate da Lodovico Frati; ricopiai schemi di canzoni inedite in manoscritti miscellanei, specie delle Biblioteche fiorentine. In questo censimento artigianale e saltuario, eseguito in margine ad altri lavori preminenti e purtroppo non più ricontrollato da allora, le diseguaglianze sono tante e di esse il Repertorio si fa specchio suo malgrado. A volte bo registrato il numero della carta in cui una canzone figura in un dato codice, a volte no: è il caso, poniamo, dell'adespota Una donzella umile e graziosa, di schema AbbCAbbC.CDdEeFfGG o AbbCAbbC.CddEeFfGG, desunta dal Riccardiano 297I. Talora il numero delle stanze (sempre aleatorio in un manoscritto, per la possibilità di lacune di trasmissione o anche per tagli consapevoli del compilatore) è indicato, talaltra no, e così il congedo, che per sua natura è un ingrediente oltremodo caduco. Oltre ai materiali suddetti, succintamente menzionati nella bibliografia, il Repertorio, in vista della pubblicazione e per scrupolo di completezza, ha registrato gli estremi di edizioni uscite anche molto tempo dopo la confezione materiale delle schede. E ha preso in conto edizioni rimaste allo stato di dattiloscritto, come quella delle Rime di Bernardo Cappello nella tesi di laurea di Enrico Albini, di Antonio Cornazano in quella di Andrea Comboni, l'Angelo Di Costanzo di Silvia Longhi, il Berardino Rota di Luca Milite [ora edito dalla Fondazione Pietro Bembo, Parma, Guanda, 2000], il Girolamo Muzio di Anna Maria Negri, il Domenico Venier di Angela Nuovo, integrato dalla tesi di dottorato padovana (200I) di Monica Bianco, l'Antonio Brocardo di Caterina Saletti, il Pietro Barignano di Maria Grazia Vecchio e della stessa Bianco, nonché il microfilm, proprietà di Simone Albonico (che me ne ha fornito anche gli estremi), delle rime di un ignoto cinquecentista di area milanese che si è convenuto di chiamare Anonimo BL, conservate in un esemplare a stampa della British Library. A questi amici va la mia gratitudine e ad Albini, scomparso da anni, il non spento ricordo del suo schivo sorriso. Tutti, tranne la Bianco, sono stati allievi di Cesare Bozzetti, inclusi Malinverni e chi scrive. Un nome, quello di Bozzetti, scomparso il 9 gennaio 1999, che è caro menzionare in questa prosa liminare. 
Il titolo che alfine si è scelto per il Repertorio colloca agli estremi cronologici le Origini e il Cinquecento. Non tutto il Cinquecento, ma solo buona parte di esso. Per dire, il Britonio si e il Carrafa no; tutto Sannazaro e Bembo, i Tasso e Celio Magno, non le canzoni delle nove famose antologie di poesia cinquecentesca uscite tra il I545 e il 1560. Qualcosa si è integrato rispetto alla schedatura originaria; ma (confesso) mi è mancata la voglia di operare in maniera sistematica l'inserzione del restante patrimonio poetico rinascimentale aggiungendo quanto mancava. Né ho avuto la pazienza di aspettare che giovani allievi e amici mi fornissero le notizie mancanti, come generosamente si proponevano di fare, né ho stimato indispensabile farlo. A costo di uscire monco di parte del Cinquecento, era meglio far uscire cosi come stava il censimento, se pur si doveva; in questa materia, attendere l'arrivo del più e del meglio è aleatorio quanto aspettare il ritorno del re Artù. Ė ben vero che sotto lo schema di Petrarca 206, S'i' 'l dissi mai, ch'i' vegna in odio a quella, si troveranno registrati rari esempi di puntuale imitazione cinquecentesca che mi ha segnalato Monica Bianco. ${ }^{3}$ Ma, comunque sia, non credo che il riempimento delle lacune possa sovvertire il panorama storico globale: $i$ cinquecentisti sono, in primo luogo, dei petrarchisti, fedeli specialmente all'ossequio metrico del modello, e l'accessione di altri schemi rispetto a quelli censiti risulta poco probabile. Spiace per la quantità, ma la qualità è forse al riparo da sorprese. A titolo di esempio, ecco quanto riserva la prima antologia storica, ossia le Rime diverse di molti eccellentissimi auttori nuovamente raccolte. Libro primo, Venezia, Giolito, IS45 (ristampa a cura di Franco Tomasi e Paolo Zaja, Collana Scrinium I9, Torino, Edizioni RES, 200I), che per l'occasione ho censito qui integralmente, ma non registrato nel REMCI.

Vi si annoverano diciotto can₹oni. Alle pp. 7-9, Pietro Bembo, Donna, de' cui begli occhi alto diletto, in morte della Morosina (qui sotto ABCBAC.CDEEDeFFGG). Alle pp. 37-39, Nicolò Amanio, Queste saranno ben lagrime, questi, in morte del proprio figlio Ippolito, ricalcato su Petrarca, Rvf 359 e altri (ABBA.ACcDdEE), da confrontare, con estensione a tredici versi, dello stesso Amanio, con Esce talor da que' begli occhi un foco, ABBA.ACcDDEeFF. Alle pp. 57-59, Giulio Camillo, Lega la benda negra, in morte del Delfino, da registrare sotto abCabC.cdeeDfF (Petrarca, Rvf $I 26$ e altri, anche con specializzazione funebre del metro). Alle pp. 68-72, Giovanni Muzzarelli, Mentre che voi ne' vaghi ampi soggiorni, in lode di Pietro Bembo. Rispetto all'individuo censito tra le Rime del Murzarelli sotto XLVII (ABCBAC.DEBbFDEEDFGHGhII), nella stampa giolitina la seconda stanza è lacunosa del secondo $\mathrm{G}$ e la quarta di h. Alle pp. 75-79, sempre del Muгzarelli, Italia mia, il tuo sì lungo pianto, canzone politica, censita in Rime XLVIII (AbCBaC.cDEeDdfGfG), dove è in compagnia di Petrarca, Rvf 128 e altri). Alle pp. 8I-85, dello stesso, Terreno Giove, a cui l'alto governo, in lode di papa Leone X, censita in Rime XXXV (ABCDABCED.fFEGEHHGgHII). A p. 92, Andrea Navagero, Veramente, Madonna, in me l'ardore, in lode di madonna (ABCCbA.DD). Alle 
Prefazione a un Repertorio metrico

Pp. II6-I2I, Bartolomeo Carli Piccolomini, Poscia ch'a sì leggiadro e chiaro obbietto, per nuovo amore (ABCBAC.CDEeDFGHHGFFIIy), Petrarca, Rvf 23 e altri). Alle $p$ p. I2I-I27, sempre di Bartolomeo Carli Piccolomini, È dunque vero, ahimè, che l'empio affanno, per malattia di madonna (ancora Petrarca, Rvf 23 e altri). Alle pp. 190-192, Iacopo Sellaio Bolognese, Or che 'l destin consente, in lode degli occhi, prima di un trittico di canzoni che non seguono però lo schema metrico delle cantilenae oculorum (Petrarca, Rvf 70-72), da censire sotto abCabC.cdeeDff (Petrarca, Rvf I25 e altri), nonché, alle pp. I92-I94, Poi che da gli occhi mei (ancora Petrarca, Rvf 126 e altri), e alle pp. 195-197, Quanto di voi ragiono (abCabC.cdeeDFF, variazione di Petrarca, Rvf I25 e I26, attuata anche da Amanio, Britonio, Ceresara e Gosellini). Alle pp. 226-229, Cosmo Rucellai, Ne la queta stagion del dolce oblio, dialogo con madonna (ABBA.ACcDdEE, Petrarca, Rvf $359 e$ altri). Alle pp. 239-242, Giovan Antonio Volpe, S'unqua vestita a panni or verdi or gialli, in morte (a Como) del proprio cugino, 6 stanze di schema ABCABC.cDEFDeFGG piu congedo: questa, a quanto pare, è di schema inattestato; una variante è Giovangiacomo Benalio, Hor debbo abbandonar l'usato inchiostro (nel Libro sesto, c. 72v), su AbCAbC.CdEFdEFGG: ed è schema che andrebbe aggiunto al REMCI tra le stanze di is versi. Alle pp. 264-265, Girolamo Muzio, Donne gentili, Amor vuol ch'io favelle, indirizzate a donne su Amore (AbCAbC.cDdEE, Petrarca, Rvf 268 e altri). Alle pp. 258-270, Ottaviano Salvi, Già per tornar vicino al nostro polo, «canzon mia amara» su primavera (ABCBAC.cddEEFeF, Petrarca, Rvf so e altri). Alle pp. 280-284, Bernardino Daniello, Sacro Signor, che 'l grand'uffizio avete, in lode del pontefice regnante, da confrontare con l'omometrica Sacro Signor, che da' superni giri del Rainerio, che si legge attribuita anche al Molza (ABCBAC.CDEEDdFF, Petrarca, Rvf 53 e altri). Alle pp. 318-321, Giovan Battista Susio, Se mai, Musa, aspre note, in morte di Marco Grimani patriarca di Aquileia (ancora ABCBAC.cddEEFeF, Petrarca, Rvf so e altri).

Questo lo specimen, sobriamente commentato, della prima antologia giolitina curata da Lodovico Domenichi, una delle crestomazie di poesia più stimate. Beninteso, bisognerà mettere in cantiere per il futuro un Repertorio tutto cinquecentesco, o (meno bene, a parer mio) una riedizione di questo, completa per il XVI secolo. Il lavoro collettivo di scelta e di commento fatto in servizio di un'antologia di poesia cinquecentesca che è in corso di elaborazione, stampato il primo tomo, ${ }^{4}$ e le eccellenti ripoposte editoriali soprattutto delle edizoni RES, fanno sperare che tale proposito sia abbastanza realistico.

Va giustificata anche la struttura del Repertorio, che si differenzia in più punti da strumenti analoghi collaudati in area romanza o specificamente in àmbito italiano, per singoli secoli o scuole il cui canone è storicamente limitato nel tempo. Penso in particolare, per le nostre lettere e per quel che attiene alla morfologia, al Repertorio metrico di Adriana Solimena per le rime dello Stil Novo (1980) e dei Poeti siculo-toscani (2000), di Roberto Antonelli per la Scuola poetica siciliana (1984), di Andrea Pelosi per la canzone del Trecento (I99I), associati a vigorosi profili storiografici, e ai contributi di Guido Capo- 
villa sulla morfologia della ballata trecentesca e del madrigale antico $(1978$ e 1982). Nonché alle classiche opere di István Frank, Répertoire métrique de la poésie des troubadours (1953-1957), di Ulrich Mölk e Friedrich Wolfzettel, Répertoire métrique de la poésie lyrique française des origines à I 350 (1972), al Repertorio metrico della lirica galego-portoghese (1967) di Giuseppe Tavani, che come i primi due studiosi citati è di scuola romana, nella quale fu decisivo il magistero, anche in materia metrica, di Aurelio Roncaglia. Maestro e pioniere di questo genere di studi in area italiana è stato Leandro Biadene, insuperato illustratore del sonetto nel I888 (anche se il lavoro sarebbe da rifare su nuovi fondamenti): un primato che il Biadene condivide con Ernesto Giacomo Parodi, i cui classici studi sulla rima (I896, I9I3) sono caposaldi storici della disciplina. Su questa via si può citare, in tempi recenti, il Rimario trobadorico provenzale compilato da Pietro G. Beltrami e Sergio Vatteroni (1988); per la versificazione $i$ contributi raccolti in volume da Mario Pazraglia (1974) e, per la prosodia, il monumentale breviario di Aldo Menichetti, Metrica italiana. Fondamenti metrici, prosodia, rima (1993), per non dire dei molti eccellenti manuali di metrica redatti in questi ultimi anni e destinati alla scuola.

Tutti questi strumenti conservano appieno la loro validità, e ad essi ci si dovrà pur sempre affidare per la parte che è la loro. Essi inoltre hanno un capitale vantaggio, che il presente Repertorio non potrebbe rivendicare a sé: per il settore limitato che è il loro, tendono alla completezza delle testimonianze. Il REMCI non è affatto un repertorio dei repertori. Nasce autonomamente da essi, magari con sue proprie lacune, dato che si è costituito man mano a partire dai testi editi di autori di quel dato tempo (e difatti ai testi e alle loro edizioni correnti si riferisce in maniera costante). E possibile che schemi di canzoni adespote, specie dei cosiddetti Siculo-toscani duecenteschi, siano sfuggiti alla rete. Non direi schemi di Siciliani in senso stretto, per $i$ quali si è spogliata integralmente la silloge di Bruno Panvini.

Tutti i repertori citati più sopra hanno adottato nella classificazione degli individui un criterio rigorosamente alfabetico: per cui si va, esemplificando sommariamente, da fronti aabaab, o anzi, trascurando le rimalmezzo, aaabaaab (che è il terzo schema di Antonelli) fino a abcdabcd, lettere che designano versi di varia misura sillabica, precisata di volta in volta da un esponente numerico. E un criterio di ordinamento che privilegia la struttura dello schema metrico, segnatamente della fronte, su altri parametri. L'ordinamento che sta alla base del REMCI segue il criterio di successione alfabetica (dove a minuscola precede A maiuscola, indicando, salvo contrario avviso, settenario vs endecasillabo) solo all'interno di stanze con lo stesso numero di versi. Nell'elenco, le canzoni si susseguono ripartite a norma della loro consistenza, da quattro a trenta versi (in tutto, sono più di millecento gli schemi 
censiti). Perché questa difformità rispetto agli strumenti consueti, e dunque alla norma invalsa?

In primo luogo bisogna dire che il mio censimento è nato così. Una volta fatte e ordinate parecchie centinaia di schede, alcune delle quali ospitano decine d'individui, non era economico ricomporne la successione con altro criterio. Questa però non è che una circostanza pratica: rispettabile certo, ma che non dà conto della scelta prima da me operata di frapporre un compartimento stagno tra canzoni (e dunque stanze) di diversa consistenza per numero di versi. A questa stregua, lo schema della 'petrosa' di Dante Io son venuto al punto de la rota (Rime C), cioè ABCABC.CDEeDFF, di tredici versi, che vige - o piuttosto è anticipato, a norma della cronologia accreditata (il caso $\grave{e}$ isolato in Dante) - anche in La dispietata mente, che pur mira (Rime $L)$, non è contiguo a quello, pure dantesco, di Gli occhi dolenti per pietà del core, ABCABC.CDEeDEFF (Rime XXV), di quattordici: eppure la fronte delle due «distese», costruita su piedi identici di tre versi, è la stessa; e la sirma - identica nella sequenza dei primi cinque versi, $\mathrm{CDEeD}$, e nella combinatio finale $\mathrm{FF}$ - è incrementata in Gli occhi dolenti di un solo endecasillabo $\mathrm{E}$ in terzultima sede, portando le rime $\mathrm{E}$ da due a tre occorrenze nel secondo esemplare. Tale affinità non è immediatamente perspicua nella struttura del Repertorio che si è adottata e qui si propone. Ma che si tratti di una semplice convenzione e non di una chiave euristica impreteribile si documenta bene con un esempio. Che dire allora della lontananza (che sarebbe malagevole superare nei repertori tradizionali) dello schema di Gli occhi dolenti da quello, cosi affine, di In quella parte dove Amor mi sprona del Petrarca ( $\operatorname{Rvf}$ I27), cioè ABCBAC.CDEeDeFF, di quattordici versi? $L a$ diversa fronte - $\mathrm{ABCABC}$ nel primo caso, $\mathrm{ABCBAC}$ nel secondocondanna $i$ due individui a non incontrarsi, nonostante la loro palese affinità morfologica, con sirma che è rispettivamente $\mathrm{CDEeDEFF} e \mathrm{CDEeDeFF}$. Affinità che è indizio di derivazione, dato che lo schema del Petrarca è certamente ricavato da architetture dantesche. Sul piano delle agnizioni non esiste a priori un parametro ideale, più utile e migliore di ogni altro. Si tratta solo di convenzioni.

Resta da vedere se quella qui adottata, conseguenza di una scelta operata alla fine degli anni sessanta, quando (a rigore) solo il Répertoire di Frank era disponibile e faceva testo, abbia senso, considerato lo scarto da una pratica che è malagevole ignorare senza buone ragioni. Confido che questo sia il caso. Va detto che la mia tesi di laurea verteva su un poeta del Cinquecento, Antonfrancesco Rainerio,' e che le prime schede censivano lirica d'imitazione petrarchesca, dal Petrarca al Tasso, lasciando da parte i duecentisti: ma piu Tre e Quattrocento, che il Cinquecento. Non era solo una scelta di comodo. La lirica illustre italiana consolida la sua morfologia una volta per tutte dopo $i$ Rerum 
vulgarium fragmenta, elaborando anche schemi inattestati, ma senza staccarsi più da quel modello. La polivalenza tipica del nostro Duecento, tanto aperto alle sperimentazioni e non di rado incline a dissimmetrie di struttura, più che con Dante ha fine col Petrarca, che fonda una duratura grammatica delle forme metriche. Endecasillabo e settenario ingredienti esclusivi della testura; fronti in prevalenza simmetriche, divisibili in moduli di quattro, sei o otto versi (solo Verdi panni [Rvf 29], con stanze di sette e due rimalmezzo, deroga a questa costante); sirma più estesa della fronte (una norma che non è applicata dal Petrarca solo in sei canzoni - Rvf II9, I35, 268, 270, 325, 360sulle ventinove raccolte in $\mathrm{Rvf}$ ) sono maniere che si affermeranno come regole. In questa morfologia della canzone, ${ }^{6}$ più della struttura della fronte conta l'ampiezza della stanza, prerequisito di una sintassi complessa, e insomma la dominante è il numero dei versi nella strofe. Il manierismo petrarchesco esige strutture più o meno ampie, subito in pronto e facilmente riconoscibili. A proposito di una canzone del Quattro o Cinquecento, la prima inchiesta o domanda da fare è: di quanti versi si compongono le stanze? Alla stessa stregua (sia detto per inciso) anche per il capitolo in terza rima, che ba struttura fissa, l'elemento determinante è la lunghezza. Nella nostra poesia questa formalizzazione si collega anche al successo del sonetto con funzione di stanza isolata di canzone. Quattordici versi viene ad essere la misura aurea di un discorso sintatticamente bene ordinato e in sé concluso, e la stanza di canzone ne resta condizionata. Non dico che ultra citraque nequit consistere rectum, ma strutture più estese di questa risultano poco maneggevoli, riservate di norma a temi gnomici, ragionativi, non lirici in senso stretto. In effetti, stanze con ampia escursione di versi hanno corso soprattutto nel Trecento. E l'esperienza insegna che stanze più estese di quattordici o quindici versi sono a rischio di una perdita di figura senza riparo e di errori d'autore, dai quali non andrà esente neppure il Tasso. Solo Guittone sembra muoversi senza disagio nelle interminabili filze di rime che uno sviluppo abnorme della stanza comporta. L'occhio non controlla più le ricorrenze, l'attesa di una data cadenza è annullata dal periodo troppo dilatato in cui s'iscrive. Si vedano ad esempio le canzoni con stanze di venti versi. La formula di Nel dolce tempo, la cosiddetta canzone delle metamorfosi (Rvf 23), perfettamente compartita e perciò memorizzabile senza difficoltà da parte di imitatori e lettori, è l'unica che conservi una riconoscibilità formale. Fu ripresa pedissequamente da più rimatori, o variata di poco, come fece Celio Magno (di cui si veda almeno Giacea presso al suo fin languida e vinta, ABCBAC.CDEeDFGGFfHH).

Si avverta che non sono state inventariate le sestine, che pure canzoni sono. Né si sono registrate le rime di ogni canzone, il che aurebbe portato il Repertorio - che conta non già duecento, ma oltre duemila capoversi, e che 
Prefazione a un Repertorio metrico

non si estende dalle Origini al 1350, ma fino al tardo Cinquecento - ad avere una mole mal compatibile con la stampa. Nella descrizione degli schemi metrici si dànno, come è noto, oscillazioni nell'uso corrente. Non si è affermata una chiara norma né per la descrizione del congedo (co.), qui definito con lettere dell'alfabeto da $\mathrm{Z}$ a ritroso, né per le rimalmezzo: $\left(\mathrm{f}_{5}, \mathrm{f}_{8}\right) \mathrm{G}$, $\left(\mathrm{f}_{5}-\mathrm{f}_{3}\right) \mathrm{G}$, o che altro? Un eccesso di formalizzazione e di segnaletica per evidenziare certi fatti (poniamo, le rime irrelate) rischia di appesantire la resa grafica senza garantire l'immediata perspicuità: in proposito, o ci si è rassegnati al silenzio, o si è redatta qualche chiosa in calce allo schema in questione. Un problema delicato riguarda l'indicazione di paternità che figura sotto $i$ capoversi. Data l'eterogeneità dei materiali messi a profitto per il censimentodall'inedito, al testo letto in un manoscritto prima ancora che in una stampa, alla stampa antica e filologicamente poco sorvegliata, alle edizioni modernel'indicazione di paternità non è mai perentoria, tranne che per le edizioni critiche e per le stampe d'autore. Va presa dunque con beneficio d'inventario, tenendo conto che una semplice etichetta non poteva farsi carico di questioni oltremodo complesse. Si è cercato di evitare un uso corrivo della formula di rima «dubbia» o «adespota», quando su certi testi si stendesse l'ombra di qualche dubbio, pì̀ o meno fondato (ma per i Siciliani ha fatto testo il criterio molto restrittivo adottato da Panvini nella sua silloge). Ci pensi il filologo a risolvere la questione: il metricista bada soprattutto a fornire schemi e notizie utili a individuare un dato testo.

Ho discorso fin qui del censimento su cui è esemplato il REMCI come di cosa mia, strumento d'uso privato ricco di dati inediti messo a disposizione di altri. L'edizione del tutto ha significato non solo trascrizione delle schede a mano su un adeguato supporto, ma anche revisione del materiale, verifica di schemi, capoversi ed edizioni, cura di uniformare e seriare secondo una corretta successione il gran numero di schede. Schede sulle quali, nel corso degli anni, si erano depositate varietà d'inchiostri, grafie e modi di citare; intermittente era stata la diligenza di registrazione, più o meno vigile nel tempo: la stessa trascrizione degli incipit sotto un medesimo lemma metrico soggetta ai più vari accidenti. Una somma di dati in cui l'estensore materiale delle schede poteva anche ritrovarsi, ma che sarebbe stato indecente riprodurre a stampa in quella veste.

Ora che mi sono staccato dal mio schedario, che non di rado contiene notizie residue ancora utili, omesse come non pertinenti, contemplo nella diversità dei formati e nella varietà delle scritture un punto di partenza superato dalla trascrizione in pulito. Che nella nuova veste non poteva esser solo cosa mia. Ho voluto che al mio nome fosse affiancato, per le cure editoriali, anche quello di Massimo Malinverni, specialmente addetto alle questioni informati- 
che. In questa fase, è stato Malinverni lo stimolo a incrementare il censimento (non solo per Panfilo Sasso e la rimeria intorno a lui, di cui è esperto riconosciuto, ma anche per altri cinquecentisti minori) e gli scarni riferimenti bibliografici, e io semmai il freno. Sentimentalmente legato com'ero al vecchio schedario, dopo tutto non mi spiaceva che l'edizione ne rispecchiasse la fisionomia talora approssimativa, quello che d'incondito, anacronistico e intempestivo inerisce a prodotti del genere, destinati a un uso privato. Il meglio poteva rivelarsi nemico del bene e ritardare in modo intollerabile $i$ tempi di stampa. Quello che si presenta al pubblico è dunque un compromesso tra artigianato di base e produzione in serie, che esige requisiti di trasparenza e di conformità. I tempi di confezione del volume sono stati assai lunghi e l'intermittenza della lavorazione non ha giovato. La trascrizione delle schede iniziò nella primavera del 1990, al tempo in cui piu attivamente operava l'Archivio della Tradizione Lirica, nel quadro dell'Istituto di Studi rinascimentali di Ferrara diretto da Amedeo Quondam e poi da Gianni Venturi, e si giovò dell'appoggio di quell'istituzione. Ha avuto da ultimo il sostegno della Fondazione Barbier-Mueller di Ginevra, sfruttandone altresi il cospicuo patrimonio di cinquecentine. Il progetto ba avuto infine un decisivo impulso a opera dell'editore Franco Cesati, che ha creduto in questo libro, che nel passaggio dal regesto manuale alla confezione per la stampa ba conosciuto momenti difficili.

Resta intatta la ragione prima che mosse a pubblicare il censimento, cioè il desiderio di mettere a disposizione di un'utenza più larga uno strumento utile agli studi di filologia italiana. Pur con le inevitabili imprecisioni e lacune, confido di non aver fallito lo scopo. ${ }^{7}$ Avrei potuto conservare le schede presso di me, giovandomene io solo per le mie ricerche: e confesso che nei momenti più ardui di confezione del volume ho deprecato di non essermi attenuto a questa condizione d'inedito, che durava da anni. E prevalso un intento, mi piace credere, meno egoista. E si sarebbero potuti sopprimere riferimenti lacunosi che fanno capolino qua e là; taluni elementi troppo vaghi, depositati sulle schede, sono risultati indecifrabili anche a me, dopo tanto tempo. Tuttavia si è preferito conservare le rare lacune, che qualche volonteroso a suo tempo potrà riempire, e dichiarare onestamente $i$ casi d'incompletezza o di fonte irreperita, piuttosto che schivare punti critici della trascrizione, privando gli utenti di dati nuovi e di prima mano. A suo tempo, non si disponeva dei quattro volumi dell'Incipitario unificato della poesia italiana (IUPI, 1988 , 1990 e 1996), né esistevano le Concordanze della Lingua Poetica Italiana delle Origini (CLPIO), uscite a stampa nel I992: $i$ riferimenti a questo capitale strumento sono stati aggiunti in seguito. L'integrazione mentale di altri dati, editoriali e filologici, è la parte attiva che si richiede al fruitore del REMCI. Si avverta che il Repertorio non fa testo se non per sommi capi per la descrizione metrica dei singoli individui (che non di rado, peraltro, risulta 
arricchita rispetto a quanto si registra nelle edizioni di riferimento), ma si vorrebbe invece affidabile per documentare la fortuna di un dato schema nel tempo. Raccolta di schemi incarnati in singoli testi. Naturalmente un Repertorio metrico dovrebbe essere idealmente perfetto, come lo è quel capolavoro di filologia in atto che è un annuario del telefono: indirizzi e numeri telefonici si susseguono senza errori, come ognuno constata a suo profitto.

Inoltre, per non fornire un prodotto esoso e inutilmente ripetitivo di dati meglio reperibili altrove, accumulando una serie di notizie mal conciliabili con la quantità e con la maneggevolezza generale del Repertorio, si è rinunciato a dar conto di molte fattispecie. Vorrei fare, a questo riguardo, una professione di fede o di gusto. La raccolta integrale dei dati è un'operazione encomiabile e preziosa, ma per sé non può avere l'efficacia di un giudizio o di una diagnosi esplicita, anche se compendiosa: diagnosi e giudizio a cui lo studioso di metrica non potrà sottrarsi con l'addurre a pretesto la completezza della sua raccolta. In nota agli schemi, si è cercato di formulare responsi epigrafici, senza tediare il lettore con liste interminabili di appunti e notizie.

L'esempio più caratteristico di una complessità metrica che nella pura rappresentazione geometrica dei fatti può risultare deviante è quello di Chiaro Davanzati. Con la sua sessantina d'individui, Chiaro si conferma come il principale produttore di «distese» della tradizione lirica antica: un vanto che egli condivide con Guittone e, all'altro estremo cronologico, con l'ineguagliabile Tasso. La metrica delle canzoni di Chiaro è notoriamente accidentatissima: si riscontrano variazioni di misura sillabica da una stanza all'altra e perfino varianti di schema delle stanze entro uno stesso individuo pluristrofico, fatti che a gran torto la filologia positivista cercò di ridurre a una misura univoca e coerente. Per non dire dei legami intermittenti tra strofe e strofe o entro la strofe stessa; o di rime designate, poniamo, $\mathrm{D}$, che riprendono puntualmente (in uno o più casi) l'uscita di rime precedenti, o della varia qualità e natura delle rime, la cui indicaz̧ione è il pregio dei repertori settoriali citati più sopra, e cosi via. Non era possibile dar conto qui di tutto questo in maniera sintetica adeguata, data l'estensione del Repertorio: per certa casistica, ci sarebbero voluti schemi speciali e una lunga prosa esplicativa di contorno. Fortunatamente per Chiaro si dispone dell'edizione critica di Menichetti, affidabile anche in questo, e ad essa senz'altro si rinvia. ${ }^{8}$ Se talvolta si sono menzionate più distesamente (anche se a intermittenza) siffatte particolarità e accidenti, ciò è accaduto soprattutto per testi che non si giovano di note metriche altrettanto sofisticate:" come, tanto per fare un esempio, Amor mi sforza e mi sprona valere di Dino Compagni (ABBACDDC.eeFfE, di tredici versi). Non si chieda a questo Repertorio una descrizione esaustiva degli individui, che si è voluta invece succinta ed essenziale. Si concede volentieri che, per il Duecento, la registrazione della pura e semplice morfolgia delle canzoni, senza che si 
forniscano informazioni sulle rime (specialmente sui legami tra le stanze, coblas capfinidas $o$ capcaudadas, $e$ sulle rime ripetute, parametri che mi sembrano particolarmente significativi), è insufficiente e quasi non ha senso. Ma la sede per una trattazione compiuta dei fatti metrici e formali in genere $\grave{e}$ elettivamente il commento ai testi: basti citare al riguardo le minuziose osservazioni metriche redatte da Antonelli nella sua edizione delle Poesie di Giacomo da Lentini (I979), a cui è d'obbligo rinviare.

Sui molti difformi individui prevale la legge statistica del genere e della specie, considerati su una durata che abbatte i confini tra secolo e secolo e consenteponiamo - di verificare quanto è ancor vivo della metrica dello Stil Novo nel Petrarca, o della maniera del Saviozzo nel Quattrocento, o soprattutto del Petrarca fino all'età del Tasso e del Marino. Per altro verso, sarebbe ingiusto tacere che molte note apposte a singoli schemi sono un contributo filologico magari solo abbozzato, ma originale e nuovo rispetto alla fonte. Basti citare, tra le canzoni con stanze di diciassette versi, $\mathrm{O}$ sacre Muse attribuita a Torquato Tasso (ABCABC.CDEFFEDdEDD), o dell'acrostico che si rintraccia (come in altri casi, non riconosciuti dagli editori) in una canzone di Antonio da Ferrara, Al cor doglioso (ABbCBCcA.ADdEFEFGG).

In linea di massima, con eccezioni debitamente segnalate, lo schema censito è quello della prima stanza: la quale però può essere affetta da lacune di trasmissione o sfigurata da errori d'autore. E quanto si verifica, poniamo, in L'aquila altiera e gloriosa insegna, che si legge sotto il nome di Alessandro Sforza (ABCBAC.CDeEDEFdF). Le stanze sono di quindici versi, ma la prima è di quattordici, a norma dell'edizione Cocito, che non avverte della lacuna. Il metricista che opera queste constatazioni non è mai disgiunto dal filologo: e per canzoni pluristrofiche con stanze di morfologia complessa (dai quindici versi in sù) più agevolmente si sospetta un errore, licenza o variante d'autore, che una lacuna della tradizione.

Inoltre, come è noto, in molte canzoni l'ultima stanza serve da congedo, modulata in formule stereotipe di commiato. Non se ne è data però speciale avvertenza. Il congedo è menzionato solo quando figuri con una sua propria struttura, di norma inferiore alla misura della stanza. Il segno dieretico non viene registrato, né le virgolette di un discorso diretto che si apra nel capoverso, né integrazioni segnate, nelle edizioni di riferimento, tra parentesi quadre, o uncinate, o con lettere stampate in corsivo (del tipo $\operatorname{vogl}<\mathrm{i}>\mathrm{a}, \operatorname{ag}[\mathrm{g}] \mathrm{io}$ ), a meno che l'intervento non muti la consistenza sillabica del verso, o la sua seriazione secondo l'alfabeto (cosi [Ma]donna o [Un] novello). Anche le lettere greche del Trissino, con buona pace di un cosi scaltro artefice, sono state sacrificate alla legge della maggioranza e di una più generale leggibilità. La distinzione tra fronte e sirma, non sempre perspicua (basti citare il caso del 
Palmario, che presenta fronti con numero di versi dispari, irriducibili a schemi sicuri), si è effettuata ponendo nello schema un punto fermo tra le parti.

Per Michelangelo, autore di componimenti monostrofici che è incerto se classificare come madrigali o stanze isolate di canzone, si è preferito abbondare, producendone tutti gli schemi nel Repertorio. Ma per questa via, testi di analoga fisionomia, che comunemente s'interpretano come madrigali (ad esempio, Palma leggiadra e viva di Galeazzo di Tarsia), sono stati esclusi, e altri potevano esservi ammessi, di Giovan Battista Strozzi o del Tasso. Non si è dato però seguito a questa ipotesi. Ci si contenta di segnalare la necessità di un compiuto regesto metrico del madrigale cinquecentesco, che qui è fuori questione realizzare. Col senno di poi, direi che stanze isolate di canzone sono proponibili solo nel Duecento (tranne che si tratti, specie nel Cinquecento, di puntuali recuperi arcaizzanti) e che è meglio ipotizzare al riguardo la confezione di veri e propri madrigali. Anche in questo caso, piuttosto che mutilare il censimento di un certo numero d'individui in omaggio a una coerenza astratta e tutta da verificare, si è preferito censire queste ambigue forme madrigalesche, tanto per una prima documentazione del fenomeno.

Con vero rammarico non si sono trascritte, se non in pochi casi, certe didascalie dei manoscritti, preziose per indicazioni di genere o altre: così, ad esempio, Chi te contrista ne la età fiorita del Boiardo (Rime CLI) è per l'autore un "Dialogus cantu isdem desinentiis respondente versibus rithimis conversis», ma è chiosa che si leggerà, con altre latine apposte ad altri componimenti, nell'edizione di Mengaldo, o in quella commentata (1998) o critica (2002) di Zanato. E per le effuse didascalie del codice Isoldiano, anch'esse notevoli, è indispensabile ricorrere alla vecchia stampa del Frati. Del pari incresciosa l'impossibilità di dar conto di titoli o denominazioni d'autore che si leggono nel congedo o in altri punti del testo. Cosi per tre canzoni di Dante, la "Contrali-erranti〉 del quarto trattato del Convivio (Rime LXXXII I4I), la «montanina» Amor, da che convien pur ch'io mi doglia (Rime CXVI 76) e già Gli occhi dolenti per pietà del core, la «cattivella canzone» di Vita Nova 20 [XXXI] 3 (cosi denominata nella prosa, ma nei versi «Pietosa mia canzone», v. 7I, e «figlinola di Tristitia», v. 75). Antonio da Ferrara - un esempio tra tanti - chiama «Disperata» (v. 76) la sua canzone Amor, con più sospir conven ch'io canti (ABbCDEeF.fGHhGII); «Lamento» (v. I27) I' ho già letto el pianto di' Troiani (ABCABC.CDdEeffEGgHH); «canzonetta mia» (v. $7 I$ ) El grave carco de la soma trista, $\mathrm{ABC}\left(\mathrm{c}_{5}\right) \mathrm{ABC} .\left(\mathrm{c}_{5}\right) \operatorname{DdE}\left(\mathrm{e}_{5}\right) \mathrm{FDF}\left(\mathrm{f}_{5}\right) \mathrm{GG}$; «canzon distesa» $(v .86)$ e «canzon distesa mia» $(v$. II $)$ Vertù celeste in titol trionfante (ABbCABbC.CDdEEFfgG) $e$ Lagrime i occhi e 'l cor sospiri amari (AbbCAbbC.CDdEeFfGGhhFII). Definizioni che il lettore diligente potrà recuperare al luogo loro. 
A cosa serve, in concreto, il Repertorio? Una serie di applicazioni è proposta qui, come esempio e invito, soprattutto per delineare una possibile tipologia d'uso.

Le due canzoni Folli pensieri e vanità di core, di dieci stanze ABbCABbC.cDdEEfFGG seguite da congedo, malamente attribuita a Dante, $e$ Non si può dir che tu non possi tutto, di sei ABbCABbC.cDDEeDDFF e congedo, composta da Pietro di Dante, ostentano una singolare affinità morfologica, rafforzata dalla comune, anche se difforme, tematica religiosa. La prima è una canzone trecentesca alla Vergine, che certo non può essere di Dante (oltre al resto, basta vedere le rime): di ciò era cosciente già il Fraticelli, che la ripubblicò senżaltro tra le apocrife. La seconda, "Satira mia canzon» (v. I03), si rivolge a Dio con fiera ansia di giustizia, invoca la vendetta divina sui contemporanei per i loro vizi e ben si addice al figlio di tanto padre. Folli pensieri sarà anche, come vuole il Fraticelli, "prosa poveramente e malamente rimata»: ma la struttura metrica, elaboratissima e di gran respiro, e la stessa prolissità, invisa all'editore ottocentesco, non sono affatto disprezzabili. E che una preghiera alla Vergine si componga di dieci stanze è un'eleganza che fa le sue prove anche nel Petrarca di Vergine bella, che di sol vestita (Rvf 366), ABCBAC.CddCEf $\left(\mathrm{f}_{5}\right)$ E, richiamandosi a una tradizione letteraria illustre. ${ }^{{ }^{\circ}}$ L'indubbia apocrifia dantesca non deve deprimere un testo di discreta fattura, che anche la metrica evidenzia.

Fortuna del verso sdrucciolo, da Fazio al Saviozzo. Le canzoni Ahi donna grande, possente e magnanima, cinque stanze firmate da Fazio degli Uberti, e Valor move con senno qui principio, «estravagante» di Simone Serdini da Siena detto il Saviozzo, di sei e congedo, sono costruite, come un'altra di Niccolò Soldanieri, sullo schema ABbCACcB.BDEeDFF, testura che appare tipicamente ed esclusivamente trecentesca. A stringere $i$ rapporti tra Ahi donna grande, che è una lirica amorosa, $e$ Valor move, canzone precettiva per signori, rettori e governanti, che ne è un'accorta imitazione volta al politico, si dà la comune caratteristica di parole rima tutte sdrucciole. Affine morfologicamente a queste due è un'adespota amorosa del Trecento, Subbitamente Amor con la sua fiaccola, di cinque stanze ABbCABbC.CDEeDFF con congedo, conservata dal manoscritto Beinecke Phillipps 8826, ${ }^{\mathrm{II}}$ di provenienza pisana, che contiene una silloge coerente e di notevolissimo rilievo, nella quale, si direbbe, Fazio fa scuola. L'identità o la non illusoria affinità degli schemi metrici comporta talora, come si vede, altre riprese di lingua, di stile o di contenuti. L'impiego del verso sdrucciolo in sedi che non siano la bucolica quattrocentesca, la poesia fidenziana o $i$ libretti d'opera, va segnalato con riguardo, messo in opera in esemplari non ancora ben studiati. ${ }^{{ }^{2}}$ La segnalazione di parole sdrucciole in rima (purché adibite siste- 
maticamente) è operata nel Repertorio da chiose ad locum, dato che si è rinunciato a fornire una rappresentazione grafica del fatto, troppo onerosa da realizzare in modo perspicuo per le molte fattispecie di cui farsi carico. Anche qui il comun denominatore di un'identica morfologia prevale sulla specificità degli individui. Ciò vale anche, poniamo, nel caso della canzone-frottola del Petrarca, Mai non vo' più cantar - com'io soleva (Rvf I05), la cui complessità interna (segnatamente, di rime) non è rispecchiata qui come è possibile e conveniente fare in sede monografica. ${ }^{3}$ Le peculiarità taciute si riproducono anche nei testi che ne derivano, censiti sotto (a) $\mathrm{B}(\mathrm{b}) \mathrm{C}(\mathrm{c}) \mathrm{D}(\mathrm{a}) \mathrm{B}(\mathrm{b}) \mathrm{C}(\mathrm{c}) \mathrm{D} \cdot(\mathrm{d}) \mathrm{E}(\mathrm{e}) \mathrm{F}\left(\mathrm{f}_{\mathrm{s}}\right) \mathrm{EeF}(\mathrm{f}) \mathrm{GHhG}$.

Si può dire che l'identità di schema si associa non di rado a riprese più estese dal modello, di tema o di capoverso. La testura ABCBAC.cddEEFeF, che è della petrarchesca $\mathrm{Ne}$ la stagion che 'l ciel rapido inchina ( $\mathrm{Rvf}$ 50), lascia in taluni epigoni il calco del primo verso, con ripresa fedelissima o con varianti. E si dànno, per uno stesso metro, imitazioni più sottili, entro tempi e spazi ben circoscritti, come per tre poeti del Reame vissuti tra Quattro e Cinquecento: Quando che Febo in Ariete alberga, è l'incipit di una «dispersa» del Sannazaro, Quando l'Aurora con vermiglia fronte è un titolo del Britonio, Quando Phebo dal ciel caccia ogne stella si legge negli Amori del Caracciolo. Sennonché il Caracciolo apre tutte le sue cinque stanze su Quando, il Sannazaro tre su cinque, mentre il Britonio ignora del tutto questo artificio.

Riprese di manierismi anteriori al decisivo magistero petrarchesco si dànno anche nel pieno Cinquecento. E un capitolo, questo, tutto da indagare. Un testo del Muzio addetto all'elogio di bella Donna, decimo e ultimo in una collana di canzoni di varia testura che celebrano blasons femminili, Poi che ti piacque, Amor, che l'alma mia, di schema ABCBAC.CDEeDdFF, presenta un doppio congedo. Sono due congedi distinti, non una sesta stanza di andamento irregolare che si aggiunga alle altre: due porzioni di testo modellate rispettivamente sulla sirma e su una sequenza che corrisponde agli ultimi cinque versi di essa. E un relitto guittoniano, non so come arrivato fino al Muzio. Se viene proprio da Guittone, si avverta che nell'ottavo libro della Giuntina, ${ }^{14}$ Sonetti e canzoni di frate Guittone d'Arezzo, una canzone di lui, Tutto 'l dolor, ch'eo mai portai, fu gioia, presenta due congedi. Anche Dante, a onor del vero, orna di più congedi Tre donne intorno al cor mi son venute (Rime CIV) $e$ Io sento sì d'Amor la gran possanza (Rime XCI), ma la Giuntina stampa, di Tre donne, solo il primo (Canzoni morali di Dante Alaghieri, $I V$ VI) e di Io sento sì d'Amor solo il secondo, omessa la sesta stanza che funge da tornata (Canzoni amorose e morali di Dante Alaghieri, III III). La Giuntina di rime antiche, che (sia detto per inciso) non è avversa alle teorie del Bembo, ${ }^{\mathrm{IS}}$ 
corredando di esempi rari le Prose di lui, pare confermarsi come un autorevole modello di singolarità formali nel Cinquecento bembista. ${ }^{16}$

$E$ che dire del cinquecentista che va sotto il nome di Anonimo $\mathrm{BL}$, che in una sua canzone, Se la sera contar fra me vo' l'hore, con stanze di dodici versi $\mathrm{AbCaBdEcDffE}$, mette insieme sei coblas unissonans? Ė dato ritrovare un artificio del genere tra gli imitatori pedissequi di Verdi panni, sanguigni, oscuri o persi (Rvf 29) e dell'escondit petrarchesco S'i' 'l dissi mai, ch'i' vegna in odio a quella (Rvf 206), $i$ soli modelli comunemente noti di quella maniera. O presso il giovane e dotto Lodovico Martelli (AbCDEFGh), o il dottissimo Trissino, La bella Donna a cui donaste il cuore $(\mathrm{AbCDeFGH})$, o infine, non sine quare, tra gli eserciz̨i poetici del modenese Giovanni Maria Barbieri (AbCDEFgH). Che l'Anonimo BL si cimenti in questa prova è il segno rivelatore di una maestria tecnica fuori del comune, che può servire anche da indiæio per riconoscere la sua personalità poetica, se non la sua stessa persona fisica.

Non sono esplicitate nel REMCI certe caratteristiche strutturali rilevanti, $e$ l'avvertenza che se ne dà qui valga anche da risarcimento di quel che il Repertorio non registra. Ad esempio, il verso finale di ciascuna delle cinque stanze della canzone petrarchesca Lasso me, ch'i' non so in qual parte pieghi ( $\operatorname{Rvf} 70$ ) ospita, come si sa, un capoverso famoso, a formare un ideale catalogo di auctoritates; ma solo parte degli individui raggruppati sotto ABBA.AccADD (segnatamente Berardino Rota e Torquato Tasso) si attengono a questa norma. ${ }^{17}$ Ed è noto a ogni lettore del Petrarca che le can₹oni 70-7I dei Rvf sono tre «sorelle», identiche per metro. Non sorprende dunque che Giovangiacomo Benalio e Lodovico Martelli abbiano prodotto a loro volta una terna di canzoni imitate dalle cantilenae oculorum; e se Torquato Tasso ne presenta due sole (Perché la vita è breve $e$ Perché l'ingegno perde) è perché una canzone affine ad esse, Donna gentile, io veggio, ha uno schema di poco variato nella sirma, e si trova dunque censita sotto il contiguo schema aBCbAC.CDEeDfEFF. Giuliano de' Medici, con due prove collocate una di séguito all'altra nel suo libro di rime (Se li amorosi strali $e$ Poiché per lunga prova), a quanto si presume vorrebbe tenere, ma non tiene, il passo del modello.

Lo schema di Amor, se vuo' ch'i' torni al giogo anticho $(\operatorname{Rvf} 270) e$ di Tacer non posso, et temo non adopre (Rvf 325$)$ è lo stesso, ABbCBAaC.CDEeDFF. Ripresa gemellare a distanza che autorizza il Muzzarelli dell'Amorosa Opra a fare altrettanto, mentre Girolamo Britonio e Celio Magno realizzeranno ben tre esemplari in questo schema. L'ultima canzone del Canzoniere petrarchesco, Vergine bella, che di sol vestita, in aggiunta a quanto si ricava dalle occorrenze censite sotto lo schema $\mathrm{ABCBAC} . \mathrm{CddCEf}\left(\mathrm{f}_{5}\right) \mathrm{E}$, presenta singolarità di rilievo, come la ripeti- 
zione di Vergine al primo e al nono verso di ogni stanza, che gli imitatori pedissequi non mancano di rispecchiare nelle loro prove. Perché c'è modo e modo di riproporre uno stesso schema, che solo la lettura diretta dei testi può apprezzare nel giusto grado. Sotto ABCABC.CDEeDFF, ad esempio, si registrano le due «distese» di Dante, La dispietata mente, che pur mira (Rime $L) e$ Io son venuto al punto de la rota (Rime C), morfologicamente identiche. Sennonché la 'petrosa' presenta una caratteristica che l'altra non ha: le parole rima FF della combinatio sono le stesse entro una stessa stanza (petra, donna, tempo, sempre, dolce, e marmo nel congedo). Il Repertorio esorta a una conoscenza dei testi di prima mano. Certo non la può surrogare, neppure nelle caratteristiche formali che toccano da vicino la testura.

Non pare che Lo mio doglioso core di Chiaro Davanzati, lamento per la perdita della donna amata, abbcabbc.deeddeed, $e$ Lo mio gioioso core di Neri de' Visdomini, abbCabbC.dedeffg(g)H, entrambe di autore fiorentino e su cinque stanze, siano state messe a raffronto. L'affinità di schema esalta la variatio dei capoversi, polarmente opposti, dato che si passa da un gioioso a un doglioso core. La depressione di $\mathrm{C}$ da endecasillabo a settenario nella fronte di Chiaro, nonché dell'ultimo verso della stanza, sarà in ossequio allo stile elegiaco, se ̀̀ vero che l'eptasillabo, che qui però non è incipitario, procede «non sine quodam elegie umbraculo» (De vulgari II XII 6). Naturalmente il percorso è da invertire se Lo mio gioioso core (V 92), che nel Vaticano lat. 3793 precede la canzone di Chiaro (V 247), è il testo di data più recente. Preferisco tuttavia postulare un percorso stilistico da euforico in Neri,

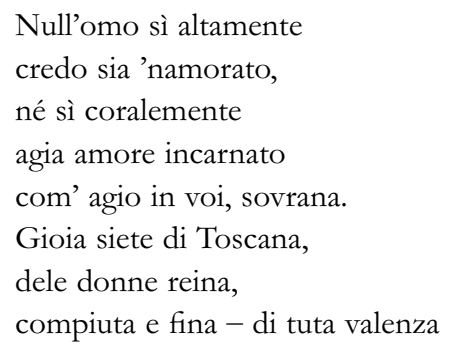

(vv. 9-16)

a disforico in Chiaro, anche a norma dell'ordinamento del manoscritto.

Siamo meno fortunati nel caso di Morte, da che convien pur ch'io mi doglia, canzone adespota e inedita (a quanto presumo), che nell'attacco varia la «montanina» di Dante, Amor, da che convien pur ch'io mi doglia. L'adespota ha schema AbCAbC.CddEE, sourapponibile al dantesco, AbCAbC.CDdECDDEE, a patto di mutilare la sirma di questo della 
sequenza CDDE, segno che la ripresa da Dante è sicura. La variante è indizio di gusti nuovi, dato che si ha ragione di ritenere che Morte, da che convien, componimento misterioso a ogni effetto, spetti al tardo Quattrocento o al Cinquecento. Il metro della «montanina» era già stato adottato in maniera pedissequa da un poeta giocoso del tempo di Dante, Neri Moscoli, perugino di Città di Castello $e$ - in una versione di soli endecasillabi $\mathrm{AB}\left(\mathrm{b}_{5}\right) \mathrm{CAB}\left(\mathrm{b}_{5}\right) \mathrm{C} . \mathrm{CDDECDDEE}$ - dal fiorentino Iacopo Cecchi, Morte, perch'io non trovo a cui mi doglia. Se s'interroga lo schema contiguo, AbCAbC.CDdEFfEGG, si vedrà che questo, con una lieve modificazione della sirma che consente di dilatare l'impiego delle rime da cinque a sette, sarà il metro per eccellenza del fiorentino Niccolò di Neri Soldanieri.

Chi è mai il Gregorio d'Arezzo, d'oscura anagrafe, che firma non meno di otto canzoni di schema ABbCABbC.CDdECDdEFF, con stanze di diciotto versi, ereditato da un poeta guelfo di parte bianca come lui, ossia dal Sennuccio del Bene di Da poi ch'i' ho perduta ogni speranza? La canzone di Sennuccio è un lamento in morte (I3I3) di Arrigo VII, che impiega il modulo tetrastico XYyZ, tipico del serventese incatenato su base quaternaria, sia nei due piedi, sia nella sirma, per due volte di seguito: una testura facile dunque, ripetitiva, che si addice a una scrittura lamentosa e insieme narrativa. Esempi di uso esclusivo e ripetuto di uno stesso metro - nella seriazione di segmenti identici, la stanza di canzone diventa simile all'ottava dei poemi narrativi, o al sonetto nella Collana o Corona di sonetti - sono anche le tre cantilenae oculorum del Petrarca (Rvf 7I, 72, 73) su aBCbAC.CDEeDfDFF, o le venti canzoni di Bindo Bonichi su abbCabbC.DEeFDEeF o abbCabbC.DEeFFEeD, entrambi con stanze di sedici versi, schema che varia di poco il precedente. Che i rimatori, nel confezionare «distese», si affezionino a un dato metro, lo si constata ancora nel pieno Cinquecento nel caso del Rota, autore di tre canzoni funebri AaBb.cdceeDfF.

In un recente contributo ${ }^{18}$ mi sono occupato, tra l'altro, di un caso di omometria $^{19}$ complicato da omonimia. La famosa canzone per «giovinetta altera» $e$ schiva d'amore, Cruda, selvaggia, fugitiva fera di Bartolomeo da Castel della Pieve, nel codice Marucelliano C. I52, autorevole testimone di rime trecentesche, ̀̀ seguita da un'adespota sullo stesso metro ABCABC.CDdEEffGGHH di diciassette, cioè Muove l'alto Fattor l'occhio beato, che costituisce l'unico caso a me noto di riutilizzo dello schema suddetto. La mano che compone $i$ due testi è dunque la stessa? Il nesso che, in uno stesso manoscritto, collega senza soluzione di continuità due testi omometrici non può essersi prodotto casualmente: una rapida inchiesta dourebbe chiarire questo caso di canzoni gemelle. Sempre a proposito di un 
autore che si chiami Bartolomeo, si dà omometria su ABBCABBC.CDdEEFfGG, di diciassette, tra Ai lacrimosa mente che t'accendi, firmata da un Bartolomeo Monaldeschi, e O sempiterna dea a cui ministra di Bartolomeo da Castel della Pieve. Questo schema caratteristico di «Bartolomeo» che cosa significa? Monaldeschi sarebbe dunque il casato di quel Bartolomeo che di norma si denomina dal luogo d'origine, Castel della Pieve, oggi Città della Pieve? Oppure O sempiterna dea è il testo di un Bartolomeo Monaldeschi distinto dal suo omonimo, che per lectio facilior applicata all'authorship (Bartolomeo da Castel della Pieve è, si presume, più conosciuto di Bartolomeo Monaldeschi) venga privato del suo prodotto? Anche in questo caso un'expertise stilistica, o meglio un'indagine storica e archivistica su Bartolomeo, non più effettuata dai tempi del Novati, che ebbe simpatia per questo rimatore, potranno chiarire la questione.

Parodia può anche comportare, nei casi più rigorosi, un calco metrico perfetto. Cosi la petrarchesca Quando il soave mio fido conforto (Rvf 359) presta lo schema, e in un caso anche l'incipit, a tre riscritture spirituali del Savonarola su ABBA.ACcDdEE, di undici. Come si vede, la strada del Petrarca spirituale (I536) di Girolamo Malipiero è già aperta nella Firenze di fine Quattrocento. E se dalla parodia si passa al manierismo, la quarantina di riprese dello schema AbCAbC.cDdEE di Che debb'io far? che mi consigli, Amore? (Rvf 268) documentano con bella evidenza, meglio di altri fatti esteriori, che cosa significhi petrarchismo nella lirica volgare. Petrarchismo cinquecentesco, perché le riprese sono tutte firmate da cinquecentisti, con la sola eccezione dell'urbinate Angelo Galli, rimatore di primo Quattrocento. Per non dire delle ottantacinque imitazioni, anch'esse in massima parte cinquecentesche, di Chiare, fresche et dolci acque (Rvf I26), di schema abCabC.cdeeDfF: Torquato Tasso vi spicca per molte coerenti riscritture, che esordiscono su $\mathrm{O}(\mathrm{h})$ e su Già. Poco meno di cinquanta le riprese della canzone gemella Se 'l pensier che mi strugge (Rvf I25), abCabC.cdeeDff, di tredici. Va segnalata, in proposito, la specializzazione matrimoniale, come di imeneo in volgare, che tocca in sorte al metro.

Nelle schede compilate a mano, $i$ capoversi delle canzoni si susseguivano in maniera casuale sotto lo stesso lemma metrico, secondo il ritmo delle letture e delle conseguenti registrazioni. In vista della stampa, i capoversi sono stati ordinati alfabeticamente, con un criterio oggettivo che ha l'inconveniente di tradire la cronologia più elementare. Ad esempio, entro lo schema ABBA.AccADD, Lasso me, ch'i' non so in qual parte pieghi (Rvf 70) del Petrarca, che è il testo più antico, capostipite di tutti quanti, è al quinto posto di tredici, preceduto perfino dal Tasso. E per ABCABC.cDEeDD, di dodici, il Petrarca, che ne è l'inventore con Standomi un giorno solo a la fenestra $(\operatorname{Rvf} 323)$, è confinato addirittura all'ultimo posto. Incongruenze 
inevitabili, posto che la cronologia, da ricostruire positivamente o indiziariamente, è una sfida per il lettore: un repertorio, di per sé, non pù surrogare l'inchiesta e il lavoro di ricostruzione dello storico, né arrogarsene le competenze. E il solo alfabeto che serve a reperire un dato capoverso: non identificato, sotto un dato schema, da un numero progressivo suo proprio, perché il corpus del REMCI è aperto, integrabile con altri individui, non già censito una volta per tutte, come i trovatori nel $\mathrm{BdT}$ o i Siciliani.

L'indagine metrica conferma l'eccellente cultura tecnica e la professionalità di certi rimatori che nelle storie letterarie hanno credito di buoni dilettanti. E il caso di Brizio Visconti, figlio naturale di Luchino, di cui restano quattro canzoni, edite tutte, forse in veste più toscaneggiante del dovuto per pregiudizio linguistico, nella meritoria antologia di poeti del Trecento approntata dal Corsi. Tre sono di schema AbCAbC.CddEeFF: Mal d'Amor parla chi d'Amor non sente, dodici stanze con congedo, illustra in modi vicini a Fazio il «tipo estetico della donna nel medio evo» (secondo la formula del Renier, che fu anche editore di Brizio); Quasi come imperfetta creatura, sei stanze con congedo, è un'apologia delle donne (si argomenta che Maria riscatta la colpa di Eva, e si appronta un compendioso catalogo de mulieribus claris che dovrebbe convincere $i$ dubbiost); Se machie molte e se ' più brutti segni, sei stanze con congedo per la morte di una «donzella» amata da Brizio, è inviata alle donne fiorentine conterranee della defunta ed è conservata dal Riccardiano 109I. Il programma filogino di Brizio è dunque metricamente coerente. E il suo schema prediletto coincide con quello di una canzone del Boccaccio, tramandata in condizioni testuali deplorevoli, Nascosi son gli spirti e l'ombre tolte, cinque stanze e congedo. La quarta canzone di Brizio, Poi ch'è cotanto menomato il vero, sei stanze ABCABC.cDdEeFfGG di quindici e congedo, coincide nello schema con una di Fazio su rime sdrucciole e con altra attribuita a Cino. Prodotti tutti di tecnica sperimentata.

Il misterioso Ciuccio, per il quale si dispone ora di un'edizione moderna e di qualche notizia supplementare ${ }^{20}$ che conferma la diagnosi continiana di un'origine e di una lingua «più meridionale della toscana, probabilmente umbras (PDuecento I 365), alla luce dello schema impiegato in [Ma]donna, eo forzirag $[\mathrm{g}]$ io lo podere, quattro stanze ABcABc.D$\mathrm{dEeFF}$, riproposto tale e quale nella «gobola» isolata Lo [meo] lontano e periglioso afanno, rivela un tratto significativo della sua fisionomia stilistica. Ciuccio riprende qui una testura del Guinizzelli, Madonna, il fino amor ched eo vo porto, di sette stanze con congedo, citata nel De vulgari $(I X V$ 6) come canzone caratteristica di Guido, «maximus» tra $i$ «poetantes Bononie [...] qui doctores fuerunt illustres et vulgarium discretione repletis. ${ }^{21}$ L'imitazione che ne fa Ciuccio è un buon indizio del prestigio riconosciuto 
fuori dell'opera dantesca al Guinizzelli, la cui stella - dalla Vita Nova a Purgatorio 26- non si appanna mai.

Singolare è il rapporto che si delinea tra il pistoiese Lemmo Orlandi ("di Iohanni d'Orlandi»), morto nel I293, e un oscuro carneade della rimeria duecentesca, Mino del Pavesaio d'Arezzo, non incluso da Contini nella sua antologia ricciardiana a differenza dell'Orlandi (PDuecento I 35I$352)$ e dunque caduto in prescrizione. Fèra cagione e dura di Lemmo e Stato son lungiamente di Mino condividono lo schema metrico aBbc.cDdEffG $(\mathrm{g}) \mathrm{HhI}$, di quattordici versi, che presenta tre rime irrelate a, E, I. Questa coincidenza è anche più sostanziosa alla luce dell'ordinamento del Laurenziano Rediano 9, nel quale Stato son (L 86) precede immediatamente due canzoni di Lemmo, Gravoso affanno e pena (L 87), il cui schema è un hapax, e Fèra cagione (L 88) appunto. Una vicinanza e una solidarietà metrica che andranno chiarite meglio in altra sede, ma che non possono essere casuali.

Parametro prezioso di conoscenza, non sempre la morfologia metrica indirizza perentoriamente verso un'area o un'onomastica precisa. La canzone Quando eu stava in le tu' cathene, messa in luce e illustrata da Alfredo Stussi, ${ }^{22}$ è di cronologia tanto alta da obbligarci a riesaminare non solo la storia letteraria, ma anche quella delle forme metriche, se ̀̀ da credere che una fronte $\mathrm{ababab}^{23}$ di tutti decasillabi avesse corso nella lirica d'arte prima che il Notaro componesse $i$ primi sonetti. Quando eu stava ha schema

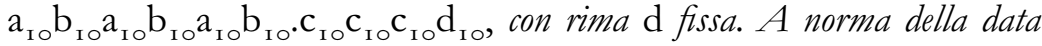
presunta della canzone, ben si comprende che l'editore privilegi $i$ paralleli metrici con testi provenzali (soprattutto produttivi risultano essere quelli con trovatori di area italiana) recuperati dal Répertoire del Frank; e che in omaggio all'ipotesi di un'origine settentrionale del testo, che sarebbe passato attraverso una trascrizione mediana («marchigiana»?), specialmente insista sulle affinità morfologiche con analoghi settentrionali, quali la canzone di Auliver, endecasillabi ABABAB.CCDD (I0.052), o le Noie di Gerardo Patecchio, otto stanze $\mathrm{a}_{10} \mathrm{~b}_{{ }_{10}} a_{10} b_{{ }_{10}} a_{{ }_{10}} b_{{ }_{10}} \cdot c_{{ }_{10}} c_{10} c_{10} c_{10}$ e congedo pari agli ultimi sei versi, con le due risposte per le rime di Ugo di Perso. Sennonché uno schema metrico affine, come non manca di segnalare Stussi, è anche nella canzone composta dal Castra fiorentino «in improperium» (De vulgari I XI 4) delle parlate centro-italiche (Roma, Marca Anconitana, Spoleto), Una fermana iscoppai da Cascioli, cinque stanze d'endecasillabi ABABAB.CDCD, nonché in testi siciliani, come La mia vit'è sì fort'e dura e fera attribuita (non bene, a quanto pare) a Guido delle Colonne, $\mathrm{ABABAB} \cdot \mathrm{c}_{8} \mathrm{dc}\left(\mathrm{c}_{4}\right) \mathrm{D}$, che peraltro ba una sirma decisamente difforme, mal comparabile con quella. Gli schemi più simili di scuola siciliana sono di canzoni il cui autore purtroppo è ignoto, Unovello pensiero ò al core e 
voglia $(V 67) e$ Già mai null'omo non à sì gra richeze $(V>I)$, sotto ABABAB.CCCD, trascritte in una sezione del canzoniere Vaticano compattamente adespota. Questa volta l'ago della bussola ruota freneticamente intorno al suo asse e non lascia scorgere all'orizzonte una stella polare che ci guidi: la metrica spinge verso Nord o verso Sud? Volentieri si sottoscrive la conclusione perplessa dell'editore, che pure è incline ad accreditare un'origine settentrionale del testo: ${ }^{24}$ "Nel prosieguo occorrerà dunque sottoporre ad accurata verifica l'opinione vulgata che colloca l'inizio della Scuola poetica siciliana nel terzo decennio del Duecento». In effetti, «un'eventuale consistente retrodatazione, purché sorretta da robuste prove documentarie, potrebbe autorizzare varie ipotesi alternative» a quella privilegiata da Stussi, ad esempio «che i versi della pergamena ravennate siano opera non di anticipatori, ma di precoci seguaci》.

Un semplice sguardo agli individui raccolti sotto 17.054 dice molto sull'ambiente, il periodo storico e la cultura formale in cui venne prodotto il portento metrico di Lorenzo da Cagli, Conctipotens eterno et summo Iddio, sessantacinque stanze ABbCABbC.CDdEEFfGG. E così, per il primo Cinquecento, la rassegna che si dispiega per lo schema ABBA.ACcDD, documentato in cinque autori del rilievo di Ariosto, Bembo, Muгio, Guidiccioni e Trissino (il capostipite della serie è, beninteso, il Bembo). Non saprei che nome fare a proposito della canzone, sicuramente trecentesca, Increata virtù del sommo cielo, che a mia notizia è inedita e si legge nel manoscritto Tempi 2 della Laurenziana: si segnala alla nostra attenzione sia perché condivide l'attacco di un sonetto di Nicolò de' Rossi, Increata vertù, eo non contendo, ${ }^{25}$ sia per la singolarità di ricalcare, essa sola, lo schema ABCABC.CDdEeCDD di Donna pietosa e di novella etate, la seconda canzone della Vita Nova.

Questa rassegna, che mostra con qualche esempio quale frutto si può trarre, oltre alle notizie morfologiche e strutturali pure e semplici, da una consultazione non frettolosa del REMCI, vuol concludersi con la menzione di un lavoro non fatto, un'inchiesta sul quattrocentista padovano Iacopo Sanguinacci. Due canzoni del Saviozzo, Addio chi sta, ch'io me ne vo cantando (Rime $L X X)$ e Perché, fuggendo il tempo, fuggon gli anni (Rime LXXI) condividono lo schema ABABCcD.DEeFfGG: la prima ba dieci, la seconda otto stanze, ed entrambe sono fornite di un congedo eguale alla sirma. Questi due testi hanno una tradizione piuttosto ridotta, a paragone del restante corpus del Saviozzo: l'unico testimone che conserva entrambi $i$ componimenti, pur separati da altri dell'autore, è il Canoniciano it. SI della Bodleian Library di Oxford, siglato $\mathrm{Can}^{2}$ da Pasquini nella sua edizione del Saviozzo. Il manoscritto oxoniense è, sia detto per inciso, un 
testimone importante anche nella tradizione delle rime del Sanguinacci, di cui purtroppo non esiste edizione critica, che vivamente si auspica. Orbene, tre canzoni del Sanguinacci, Angosce, pianti, guai, doglie e martiri, Fellice chi misura ogni suo passo (di paternità da verificare) $e$ Patre del cielo e re de gl'emisferi hanno, pur con varia estensione, lo schema delle due «estravaganti» del Saviozzo. ${ }^{26}$ E la canzone del Sanguinacci a Lionello d'Este, Non perch'io sia bastante a dechiararte, reimpiega lo schema ABbCADdC.CEeFfGG di Gloriasi 'l celeste e l'uman langue, composta dal Saviozzo come encomio funebre di Giovanni di Azzo degli Ubaldini da Firenze. Si profila un manierismo formale spiccato, e si ba una conferma della grande e perdurante fortuna del Saviozzo: presso l'eclettico Sanguinacci, non meno che in un lirico puro come Giusto de' Conti. Sennonché Sanguinacci e Saviozzo hanno in comune le prime lettere del nome, il che puì aver ingenerato confusione nelle didascalie dei manoscritti, magari per l'ambigua sigla Sa; $e$ in materia d'identità morfologiche iterate, le circostanze sono da vagliare con una prudenza tutta speciale. Questo nodo del Sanguinacci va sciolto, e la metrica aiuta a porre, se non a risolvere, ardui problemi anche in materia d'attribuzione e di canone.

Forse non è elegante confessarlo apertamente, ma la confezione del REMCI $\grave{e}$ costata al suo autore una fatica immane, più che qualunque altro lavoro: fatica aggravata dall'assillo costante del non-finito e del perfettibile. Staccarmi dalle mie schede dandole alle stampe è stato anche un atto doloroso di resa, la confessione di un limite. Chi mi è vicino nel lavoro e nella vita sa che la metrica della canzone da divertimento si è trasformata in tormento, specie da quando era disponibile una provvisoria versione a stampa, del tutto insoddisfacente nella sua prima tiratura. E a quel punto, risanato e integrato tutto quel che si è potuto, era bene sgravarsene, e cosi ho fatto. In chiusa dei lavori, mi è caro ringraziare della pazienza e dell'aiuto loro nella revisione di schede, nel controllo e reperimento di materiali rari anzitutto mia moglie Paola Allegretti. E poi Giovanni Bardazzi, Monica Bianco, Agostino Casu, Massimo Danzi, Massimo Malinverni (attivo anche dopo la conclusione della sua parte), Matteo Residori, Nelia Cacace Saxby, Fabio Zinelli e il mio bibliotecario a Ginevra Carlo Lagomarsino, solleciti assistenti della mia tela: piuttosto che di Aragne o Minerva, tela infinita di Penelope.

Guglielmo Gorni

Ginevra/Foligno, dicembre 2001/agosto 2005 


\section{Guglielmo Gorni}

Si stampa su Italique, con qualche ritocco formale, dato che tarda l'uscita in volume del REMCI presso Franco Cesati di Firenze, la sua Prefazione, particolarmente importante, io credo. La Fondazione Barbier-Mueller a suo tempo sostenne la confezione dell'opera, con un contributo finanziario a Massimo Malinverni e con la messa a disposizione del suo patrimonio librario, come si dice anche più oltre.

I. Le furie di Gadda, recensione a Carlo Emilio Gadda, Saggi Giornali Favole e altri scritti, II, a cura di C. Vela, G. Pinotti, D. Isella, G. Gaspari, F. Gavazzeni, M. A. Terzoli, Milano, Garzanti, I992, in «L'Indice», giugno I992, n. 6, pp. 8-9.

2. Ragioni metriche della canzone, tra filologia e storia, in Studi di filologia e di letteratura italiana offerti a Carlo Dionisotti, Milano-Napoli, Ricciardi, I973, pp. I 5-24, ristampato in Metrica e analisi letteraria, Bologna, il Mulino, I993, pp. 207-I 8. Di questo mio saggio e di altri, segnatamente Appunti metrici e testuali sulle Rime di Alessandro Sforza, in «Giornale Storico della Letteratura italiana», CLII (1975), pp. 222-33, si sono integrate le risultanze negli schemi metrici a cui si applicano. E altre correzioni che risultano, a mio parere, sicure, seppur non divulgate a stampa, si sono tacitamente applicate in vari casi.

3. S'altro amor seguo, i' prego 'l ciel che mai di Pietro Barignano, S'i' 'l dissi mai, che 'l ciel empio e rubello di Iacopo Zane. Non so donde viene la citazione di S'io 'l dissi per turbar quel sacro e chiaro di Angelo Di Costanzo, che trovo tra le mie schede.

4. Cfr. Poeti del Cinquecento. I. Poeti lirici, burleschi, satirici e didascalici, a cura di Guglielmo Gorni, Massimo Danzi e Silvia Longhi, La letteratura italiana. Storia e testi, Volume 23, Tomo I, Ricciardi, Milano-Napoli 200I, pp. XL-I 2 I 2.

5. Ne ho ricavato un saggio anni dopo, Un'ecatombe di rime. I «Cento sonetti» di Antonfrancesco Rainerio, in «Versants. Revue suisse des littératures romanes», numero dedicato a Prologues an $X V T^{e}$ siècle, a cura di A. Gendre e M. Jeanneret, is, N. S., I989, pp. I $35-52$.

6. Ne ho tracciato un profilo nella diacronia in Le forme primarie del testo poetico, in Letteratura italiana, a cura di A. Asor Rosa, III. Le forme del testo. I. Teoria e poesia, Torino, Einaudi, 1984, pp. 439-5 I 8. Il saggio è ristampato in Metrica e analisi cit. (si vedano, per la canzone, le pp. I $5-62)$.

7. Per comune esperienza gli errori, in materia metrica, sono molto diffusi: affliggono in varia misura tutti gli strumenti e le edizioni che si sono consultati. Neppure questo Repertorio, per quanta cura si sia spesa, ne sarà esente: chi vede più un $c$ che usurpa il posto di un $C$ in uno schema metrico? L'errore, una volta installatosi, è particolarmente insidioso. Mi rimetto all'indulgenza e alla cortesia degli utenti, che sono pregati di segnalare a chi scrive non solo le lacune riscontrate, del resto già messe in conto, ma soprattutto eventuali errori, dandone notizia a guglielmo.gorni@lettres.unige.ch.

8. Menichetti, che poteva giovarsi di un'ampia bibliografia sulla metrica di Chiaro (che annovera contributi di Gaspary, Mussafia, Casini, Biadene e Palmieri, pur eventualmente contestabili), ha fornito un'ottima descrizione delle canzoni: non ho riscontrato che un'imprecisione, $F$ non $e$ è irrelata nello schema di XXV a p 93. Resta discrezionale la segnalazione di un legame tra stanza e stanza alle pp. 89 (credo/credenza, vv. I 2-I 3), Io6 (ferire/fero [?], vv. 72-74), Io9 (fallente/fallito, vv. 24-25), I42 (membrare/Rimembranza, vv. 22-23), I 99 (ripresa della stessa rima tra i vv. 29-30), 207 e 2 I I , tra i vv. 80-8 I, da aggiungere ai molti inventariati dall'editore.

9. Non sempre filologi di alta qualità e di sicuro mestiere palesano interessi metrici, talché le loro edizioni possono rivelarsi insoddisfacenti sotto questo profilo. Chi esaminasse, ad esempio, le edizioni approntate da Franca Brambilla Ageno delle rime di Panuccio e del 
Prefazione a un Repertorio metrico

canzoniere di Franco Sacchetti potrebbe lamentare più di un'omissione in materia di morfologia e di rime (vi si indicano però con diligenza gli schemi metrici di base). Schemi che Francesco Filippo Minetti non si è dato cura d'inserire nella sua edizione di Monte Andrea, che li reclamerebbe a gran voce: una canzone come Nel core ag[glgio un foco, più che una semplice scheda, meriterebbe uno studio speciale, per districare il groviglio dell'accidentata struttura.

Io. Cfr., anche per altri tratti formali, il mio Petrarca Virgini. (Lettura della canzone CCCLXVI «Vergine bella»), in Lectura Petrarce, VII, 1987, Firenze, Olschki, 1988, pp. 201-18, e gli sviluppi operati da Maurizio Perugi, Lanfranco Cigala nell'epilogo dei «Rerum vulgarium fragmenta», in «Studi Medievali», XXXII (199I), pp. 833-4I e Numerologia mariana in due antecedenti del Petrarca: il canzoniere di Guiraut Riquier e la canzone a Maria di Lanfranco Cigala, in «Anticomoderno», 4 (1999), pp. $25-44$, sulla scorta di constatazioni sul numero dieci, elettivamente mariano, che risalgono, specie per le Cantigas de Santa Maria di Alfonso X, a un innovativo studio di Valeria Bertolucci Pizzorusso. Cfr. anche Paola Allegretti, Il «geistliches Lied» come marca terminale nel canzoniere provenzale $C$, in «Studi Medievali», XXXIII (1992), pp. 72 I-35.

I I. Sull'edizione che di questo inedito canzoniere diede Rigo Mignani nel 1974 è da vedere un'importante recensione di Claudio Ciociola, che propone tra l'altro l'attribuzione di Subbitamente a Fazio: cfr. «Studi Medievali», XVII (1976), pp. 757-75.

I2. Per il periodo che è il suo, valga il bilancio di Andrea Pelosi, La canzone italiana del Trecento, in «Metrica» V (1990), pp. 3-162, alle pp. I 52-57.

13. Per cui si veda ora, con ingrandimento da microscopio elettronico, Massimo Zenari, Repertorio metrico dei «Rerum vulgarium fragmenta» di Francesco Petrarca, Padova, Antenore, I999. E cfr. la nota che correda Francesco Petrarca, Canzoniere, edizione commentata a cura di Marco Santagata, Milano, Mondadori, 1996, pp. 488-90.

14. Sonetti e canzoni di diversi antichi avtori toscani in dieci libri raccolte. Impresso in Firenze per li heredi di Philippo di Giunta nell'anno del Signore MDXXVII Adì vi del mese di Luglio. [Ristampa anastatica, con introduzione e indici di Domenico De Robertis, 2 voll., Firenze, Le Lettere, 1977].

I s. Mi attengo alla mia interpretazione dell'impresa, consegnata a Di qua e di là dal dolce stile. (In margine alla Giuntina), ora in Il nodo della lingua e il Verbo d'Amore. Studi su Dante e altri duecentisti, Firenze, Olschki, 1981, pp. 217-42.

16. Su questo ottavo libro cfr. Flaminio Pellegrini, Nuovi studi sulla Giuntina di rime antiche, in «Rassegna bibliografica della Letteratura italiana», XXI (1913), pp. I 2-30 e Michelangelo Picone, Filologia cinquecentesca: $i$ Giunti editori di Guittone, in «Yearbook of Italian Studies», II (1972), pp. 78-101, nonché, di chi scrive, Il Dante perduto. Storia vera di un falso, Torino, Einaudi, I994, pp. 88-9I.

17. Cfr. ora, di chi scrive, Per una canzone del Tasso e altre riprese di Petrarca LXX, in Sul Tasso. Studi di filologia e letteratura italiana offerti a Luigi Poma, a cura di Franco Gavazzeni, RomaPadova, Editrice Antenore, 2003, pp. 229-42.

18. Metrica e filologia attributiva. Vent'anni dopo, in Carmina semper et citharae cordi. Études de philologie et de métrique offertes à Aldo Menichetti, éditées par M.-C. Gérard-Zai, P. Gresti, S. Perrin, Ph. Vernay, M. Zenari, Genève, Slatkine, 2000, Pp. I-I 2. In questo studio tratto anche delle somiglianze di schema con Voglioso e vago a novellar d'amore di un altro Bartolomeo, il Monacheschi (allotropo di Monaldeschi?), a norma di quanto attesta il Riccardiano I09I. Mi sono occupato, in questa sede, anche della canzone Era'n quel giorno che l'alta reina, che fu malamente attribuita a Dante, e che assegno a «un epigono di Dante vicino a Lapo». 


\section{Guglielmo Gorni}

Illustra gli influssi danteschi e dà un nuovo testo e commento Giuseppe Marrani, Un frammento della fortuna dantesca: la canzone adespota Era 'n quel giorno che l'alta reina, in «Per leggere», 5 (autunno 2003), pp. 5-24.

I 9. Per omometria intendo - con scarto rispetto alla terminologia prevalente, ma non perentoria - la perfetta identità dello schema metrico delle stanze di date canzoni.

20. Mahmoud Salem Elsheikh, Il caso Ciuccio, in «Studi di Filologia italiana», XXXVIII (I980), pp. I I-32.

2 I. Il mio punto di vista riguardo a questo passo in Paralipomeni a Lippo (1989), ora in Dante prima della «Commedia», Fiesole, Cadmo, 200I, pp. 59-79, alle pp. 7I e ss. E si veda ora specialmente Claudio Giunta, La poesia italiana nell'età di Dante. La linea Bonagiunta-Guinizzelli, Bologna, il Mulino, 1999, pp. 172-78.

22. Alfredo Stussi, Versi d'amore in volgare tra la fine del secolo XII e l'inizio del XIII, in «Cultura Neolatina», LIX (1999), I-2, pp. I-70 (con una Nota paleografica a cura di A. Ciaralli e A. Petrucci, pp. 43-49, e una Nota musicologica a cura di C. Gallico, pp. 50- 5 I): le osservazioni metriche alle pp. IO-I 2 .

23. Su questa sequenza, la cosiddetta «quasi ottava» e le implicazioni storiche che è lecito (o non lecito) postulare riguardo all'origine dell'ottava rima, rinvio alla Postilla sull'ottava e sulla terza rima, in Metrica e analisi cit., pp. 295-310, specialmente le pp. 295-30I.

24. E cita con simpatia la suggestiva ipotesi di Contini (di un Contini che ragiona qui come Rajna nelle questioni di origini) a proposito della Danza mantovana, di Gotto e di Sordello "trovatore forse anche in volgare d'Italia»: «siamo dunque sulle tracce d'un centro lirico settentrionale, affiancato ai meridionali e toscani e bolognesi, ma naufragato nel tempo» (PDuecento I 785), distinto dalla Cremona di Pateg e vicini, «più arcaica», e dalla Marca trivigiana di Auliver, «ispirata a un espressionismo più moderno».

25. Cfr. Furio Brugnolo, Il Canzoniere di Nicolò de' Rossi I. Introduzione, testo e glossario, Padova, Antenore, I974, p. I I I ( $\left.{ }^{\circ} 258\right)$.

26. In verità anche quattro canzoni che vanno sotto il nome di Giovanni de' Mantelli di Canobio detto Tartaglia, comprese tra i numeri XIII e XCI dell'edizione dei Versi d'amore di lui (ed altri), che depongono naturalmente per un'identità di mano per questa sezione. In queste prove del Tartaglia la fronte, piuttosto che su quattro, sarà su sette versi, come suggerisce il modello del Serdini e come conferma la sintassi. Resta infine da tener conto dell'avventata attribuzione a Fazio, da parte dell'Isoldiano, di Deh, muta stile ormai, giovenil core. Questa canzone e Fellice ci misura condividono la chiusa ricorrente, come di ritornello, di ogni stanza: un indizio di peso nella questione della paternità. 